\title{
Novel copolymers of styrene. 15. Halogen ring-disubstituted propyl 2- cyano-3-phenyl-2-propenoates
}

Christopher R. Savittieri, Shechinah M. Tinsley, Andrew J. Diehn, Fatema Hai, Kara E. Humanski, Eric J. Kempke, Benjamin Y. Killam, Jimmy Kozeny, Evana W. Makhoul, Molly C. Obert, Anthony C. Parisi, and Victoria C. Parrilli, and Gregory B. Kharas*

DePaul University, Chemistry and Biochemistry Department, 1110 West Belden Avenue, Chicago, IL 60614-3214

Contact: gkharas@depaul.edu

\begin{abstract}
Novel halogen ring-disubstituted propyl 2-cyano-3-phenyl-2-propenoates, $\mathrm{RPhCH}=\mathrm{C}(\mathrm{CN}) \mathrm{CO}_{2} \mathrm{C}_{3} \mathrm{H}_{7}$ (where $\mathrm{R}$ is 2-fluoro-5-methyl, 3-iodo-4-methoxy, 5-iodo-2methoxy, 3,5-dichloro, 3,4-difluoro, 3,5-difluoro, 2-chloro-4-fluoro, 2-chloro-6-fluoro, 3chloro-2-fluoro, and 3-chloro-4-fluoro) were prepared and copolymerized with styrene. The propenoates were synthesized by the piperidine catalyzed Knoevenagel condensation of ring-disubstituted benzaldehydes and propyl cyanoacetate, and characterized by CHN elemental analysis, IR, ${ }^{1} \mathrm{H}$ - and ${ }^{13} \mathrm{C}$-NMR. All the propenoates were copolymerized with styrene $\left(\mathrm{M}_{1}\right)$ in solution with radical initiation $(\mathrm{ABCN})$ at $70^{\circ} \mathrm{C}$. The composition of the
\end{abstract}


copolymers was calculated from nitrogen analysis, and the structures were analyzed by IR, ${ }^{1} \mathrm{H}$ and ${ }^{13} \mathrm{C}-\mathrm{NMR}$, GPC, DSC, and TGA. Decomposition of the copolymers in nitrogen occurred in two steps, first in the $200-500^{\circ} \mathrm{C}$ range with residue $(1-3 \%$ wt.), which then decomposed in the $500-800^{\circ} \mathrm{C}$ range.

Keywords: Radical copolymerization; styrene copolymers; trisubstituted ethylenes, cyanoacrylates

\section{Introduction}

Ring-disubstituted propyl esters of 2-cyano-3-phenyl-2-propenoic acid (PCPP), $\mathrm{RPhCH}=\mathrm{C}(\mathrm{CN}) \mathrm{CO}_{2} \mathrm{C}_{3} \mathrm{H}_{7}$ continue to attract attention as compounds with interesting properties and as comonomers for modification of commercial polymers. Similar structure to 2-fluoro-5-methyl PCPP was reported in stereoselective cascade assembling of benzylidenecyanoacetates and 1,3-dimethylbarbituric acid into (1R*,2S*)-1-cyano-5,7dialkyl-4,6,8-trioxo-2-aryl-5,7-diazaspiro[2.5]octane-1-carboxylates [1], as well as in synthesis and application of polysiloxane-supported NAD(P)H model 1-Benzyl-1,4dihydronicotinamide in the reduction of activated olefins [2]. 4-Iodo ring-substituted PCPP was involved in selective hydrolysis of 1-cyanocyclopropane-1-carboxylates leading to concise preparation of 1-carbamoylcyclopropane-1-carboxylates [3], in reaction of indandione with ethyl $\alpha$-cyano- $\beta$-arylacrylates [4], and in base-free Knoevenagel condensation catalyzed by copper metal surfaces [5]. 2-Iodophenyl derivative was used in preparation of 1-carbamoylcyclopropane-1-carboxylates via selective hydrolysis of 1- 
cyanocyclopropane-1-carboxylates [6]. 3,4-Dichloro-ring substituted ethyl ester was used in preparation of a novel $\mathrm{Fe}_{3} \mathrm{O}_{4} @ \mathrm{SiO}_{2} @$ propyl@DBU magnetic core-shell nanocatalyst for Knoevenagel reaction in aqueous medium [7], in regio- and stereo-selective synthesis of spiro pyrrolidine and pyrrolizidine derivatives [8], in Knoevenagel condensation using a PEG bridged tertiary amine functionalized ionic liquid exhibiting thermoregulated reversible biphasic behavior with cyclohexane/isopropanol [9], in synthesis of 5,6-dichloroindan-1acids and their tetrazolyl derivatives as analgesic and anti-inflammatory agents [10]. It was also used in heterocyclic synthesis of some new pyridine, pyridone, pyrazole, thiophene, fused pyrimidine and triazine derivatives via $\beta$-Amino- $\beta$-(pyrid-4-yl)acrylonitrile [11]. 3,4-Difluorophenyl substituted methyl propenoate was reported in synthesis and study of histamine $\mathrm{H} 2$ agonistic activity of arpromidine analogs via replacement of the pheniraminelike moiety by non-heterocyclic groups [12]. 3,5-Dibromo ring-substituted methyl propenoate was explored in synthesis of new phenylsuccinimide derivatives with anticonvulsant properties [13]. Applications of ethyl 2-cyano-3-phenyl-2-propenoate, and its ring-substituted derivatives include: studies of stereoselective cascade assembling of benzylidenecyanoacetates and 1,3-dimethylbarbituric acid into (1 $\left.\mathrm{R}^{*}, 2 \mathrm{~S}^{*}\right)$-1-cyano-5,7dialkyl-4,6,8-trioxo-2-aryl-5,7-diazaspiro[2.5]octane-1-carboxylates [14]; studies of polysiloxane-supported NAD(P) model of 1-benzyl-1,4-dihydronicotinamide [15]; studies of stereospecific characterization and peripheral modification of 1-(pyrrolidin-1-ylmethyl)2-[(6-chloro-3-oxo-indan)-formyl]-1,2,3,4-tetrahydroisoquinolines as novel selective kappa opioid receptor agonists [16]; synthesis and analgesic activity of 6-fluoroindan-1-carboxylic 
acid [17], as well as an investigation into the oxidation mechanism of Hantzsch 1,4dihydropyridines [18].

In continuation of our search for new monomers and copolymers of trisubstituted ethylenes we have prepared halogen ring-disubstituted propyl 2-cyano-3-phenyl-2propenoates $(\mathrm{PCPP}), \mathrm{RPhCH}=\mathrm{C}(\mathrm{CN}) \mathrm{CO}_{2} \mathrm{C}_{3} \mathrm{H}_{7}$, where $\mathrm{R}$ is 2-fluoro-5-methyl, 3-iodo-4methoxy, 5-iodo-2-methoxy, 3,5-dichloro, 3,4-difluoro, 3,5-difluoro, 2-chloro-4-fluoro, 2chloro-6-fluoro, 3-chloro-2-fluoro, and 3-chloro-4-fluoro, and explore the feasibility of their copolymerization with styrene. To the best of our knowledge, there have been no reports on either synthesis of these propenoates, nor their copolymerization with styrene [19].

\section{Experimental}

2-Fluoro-5-methyl, 3-iodo-4-methoxy, 5-iodo-2-methoxy, 3,5-dichloro, 3,4-difluoro, 3,5difluoro, 2-chloro-4-fluoro, 2-chloro-6-fluoro, 3-chloro-2-fluoro, and 3-chloro-4fluorobenzaldehydes, propyl cyanoacetate, piperidine, styrene, 1,1'azobiscyclohexanecarbonitrile, (ABCN), and toluene supplied from Sigma-Aldrich Co., were used as received. Instrumentation and analyses are reported in [20].

\section{Synthesis of Monomers}

The ring-substituted propyl 2-cyano-3-phenyl-2-propenoates (PCPP) were synthesized by Knoevenagel condensation [21] of a ring-substituted benzaldehyde with propyl cyanoacetate, catalyzed by base, piperidine.

$$
\mathrm{RPhCHO}+\mathrm{NCCH}_{2} \mathrm{CO}_{2} \mathrm{C}_{3} \mathrm{H}_{7} \rightarrow \mathrm{RPhCH}=\mathrm{C}(\mathrm{CN}) \mathrm{CO}_{2} \mathrm{C}_{3} \mathrm{H}_{7}
$$


Where $\mathrm{R}$ is 2-fluoro-5-methyl, 3-iodo-4-methoxy, 5-iodo-2-methoxy, 3,5-dichloro, 3,4difluoro, 3,5-difluoro, 2-chloro-4-fluoro, 2-chloro-6-fluoro, 3-chloro-2-fluoro, and 3-chloro4-fluoro. The preparation procedure was essentially the same for all the monomers. In a typical synthesis, equimolar amounts of propyl cyanoacetate and an appropriate ringsubstituted benzaldehyde were mixed in equimolar ratio in a $20 \mathrm{~mL}$ vial. A few drops of piperidine were added with stirring. The product of the reaction was isolated by filtration and purified by crystallization from 2-propanol. The condensation reaction proceeded smoothly, yielding products, which were purified by conventional techniques.

\subsection{Propyl 2-cyano-3-(2-fluoro-5-methylphenyl)-2-propenoate}

Yield 87\%; ${ }^{1} \mathrm{H}-\mathrm{NMR} \delta 8.4(\mathrm{~s}, 1 \mathrm{H}, \mathrm{CH}=), 8.2-6.9(\mathrm{~m}, 3 \mathrm{H}, \mathrm{Ph}), 4.3\left(\mathrm{t}, 2 \mathrm{H}, \mathrm{OCH}_{2}\right), 2.3(\mathrm{~s}$, $\left.3 \mathrm{H}, \mathrm{CH}_{3}\right), 1.7\left(\mathrm{~m}, 2 \mathrm{H}, \mathrm{OCH}_{2} \mathrm{CH}_{2}\right), 1.0\left(\mathrm{t}, 3 \mathrm{H}, \mathrm{OCH}_{2} \mathrm{CH}_{2} \mathrm{CH}_{3}\right) ;{ }^{13} \mathrm{C}-\mathrm{NMR} \delta 163(\mathrm{C}=\mathrm{O})$, $152(\mathrm{HC}=), 138,135,130,121,115(\mathrm{Ph}), 116(\mathrm{CN}), 104(\mathrm{C}=), 67\left(\mathrm{O}^{\mathrm{CH}} \mathrm{H}_{2}\right), 22$

$\left(\mathrm{OCH}_{2} \underline{\mathrm{CH}}_{2}\right), 21\left(\mathrm{CH}_{3}\right), 10\left(\mathrm{OCH}_{2} \mathrm{CH}_{2} \underline{\mathrm{CH}}_{3}\right) ; \mathrm{IR}\left(\mathrm{cm}^{-1}\right): 3100-2810(\mathrm{~m}, \mathrm{C}-\mathrm{H}), 2226$ (m, CN), 1731 (s, C=O), 1609 (s, C=C), 1293 (s, C-O-C), 858, 797 (s, C-H out of plane). Anal. Calcd. for $\mathrm{C}_{14} \mathrm{H}_{14} \mathrm{FNO}_{2}$ : C, 68.00; H, 5.71; N, 5.66; Found: C, 67.85; H, 5.75; N, 5.86.

\subsection{Propyl 2-cyano-3-(3-iodo-4-methoxyphenyl)-2-propenoate}

Yield 92\%; mp $140^{\circ} \mathrm{C},{ }^{1} \mathrm{H}-\mathrm{NMR} \delta 8.3(\mathrm{~s}, 1 \mathrm{H}, \mathrm{CH}=), 8.2-6.7(\mathrm{~m}, 3 \mathrm{H}, \mathrm{Ph}), 4.3(\mathrm{t}, 2 \mathrm{H}$, $\left.\mathrm{OCH}_{2}\right), 4.0\left(\mathrm{~s}, 3 \mathrm{H}, \mathrm{OCH}_{3}\right), 1.8\left(\mathrm{~m}, 2 \mathrm{H}, \mathrm{OCH}_{2} \mathrm{CH}_{2}\right), 1.0\left(\mathrm{t}, 3 \mathrm{H}, \mathrm{OCH}_{2} \mathrm{CH}_{2} \mathrm{C}_{3}\right) ;{ }^{13} \mathrm{C}-\mathrm{NMR}$ $\delta 163(\mathrm{C}=\mathrm{O}), 154(\mathrm{HC}=), 128,118,88(\mathrm{Ph}), 116(\mathrm{CN}), 100(\mathrm{C}=), 67\left(\mathrm{OC}_{2}\right), 56$ $\left(\mathrm{OCH}_{3}\right), 22\left(\mathrm{OCH}_{2} \underline{\mathrm{CH}_{2}}\right), 10\left(\mathrm{OCH}_{2} \mathrm{CH}_{2} \underline{\mathrm{CH}}_{3}\right) ; \mathrm{IR}\left(\mathrm{cm}^{-1}\right): 3023-2760$ (m, C-H), 2219 (m, 
CN), 1715 (s, C=O), 1605 (s, C=C), 1262 (s, C-O-C), 926, 835 (s, C-H out of plane). Anal. Calcd. for $\mathrm{C}_{14} \mathrm{H}_{14} \mathrm{INO}_{3}$ : C, 45.30; H, 3.80; N, 3.77; Found: C, 44.47; H, 3.72; N, 3.33 .

\subsection{Propyl 2-cyano-3-(5-iodo-2-methoxyphenyl)-2-propenoate}

Yield 95\%; mp 93 ${ }^{\circ} \mathrm{C},{ }^{1} \mathrm{H}-\mathrm{NMR} \delta 8.2(\mathrm{~s}, 1 \mathrm{H}, \mathrm{CH}=), 7.4-6.7(\mathrm{~m}, 3 \mathrm{H}, \mathrm{Ph}), 4.3(\mathrm{t}, 2 \mathrm{H}$, $\left.\mathrm{OCH}_{2}\right), 3.9$ (s, $\left.3 \mathrm{H}, \mathrm{OCH}_{3}\right), 1.8\left(\mathrm{~m}, 2 \mathrm{H}, \mathrm{OCH}_{2} \mathrm{C}_{2}\right), 1.1$ (t, 3H, $\left.\mathrm{OCH}_{2} \mathrm{CH}_{2} \mathrm{C}_{3}\right) ;{ }^{13} \mathrm{C}-\mathrm{NMR}$ $\delta 163(\mathrm{C}=\mathrm{O}), 152(\mathrm{HC}=), 122,115,80(\mathrm{Ph}), 116(\mathrm{CN}), 111(\mathrm{C}=), 67\left(\mathrm{OCH}_{2}\right), 56\left(\mathrm{OCH}_{3}\right)$, $22\left(\mathrm{OCH}_{2} \underline{\mathrm{CH}_{2}}\right), 10\left(\mathrm{OCH}_{2} \mathrm{CH}_{2} \underline{\mathrm{CH}} 3\right) ; \mathrm{IR}\left(\mathrm{cm}^{-1}\right)$ : 3028-2798 (m, C-H), 2223 (m, CN), 1740 (s, C=O), 1599 (s, C=C), 1250 (s, C-O-C), 883, 756 (s, C-H out of plane). Anal. Calcd. for $\mathrm{C}_{14} \mathrm{H}_{14} \mathrm{INO}_{3}: \mathrm{C}, 45.30 ; \mathrm{H}, 3.80 ; \mathrm{N}, 3.77$; Found: C, 44.78; H, 3.87; N, 4.05 .

\subsection{Propyl 2-cyano-3-(3,5-dichlorophenyl)-2-propenoate}

Yield 75\%; mp $123^{\circ} \mathrm{C},{ }^{1} \mathrm{H}-\mathrm{NMR} \delta 8.1(\mathrm{~s}, 1 \mathrm{H}, \mathrm{CH}=), 7.9-7.5(\mathrm{~m}, 3 \mathrm{H}, \mathrm{Ph}), 4.3(\mathrm{t}, 2 \mathrm{H}$, $\left.\mathrm{OCH}_{2}\right), 1.8\left(\mathrm{~m}, 2 \mathrm{H}, \mathrm{OCH}_{2} \underline{\mathrm{CH}}_{2}\right), 1.0\left(\mathrm{t}, 3 \mathrm{H}, \mathrm{OCH}_{2} \mathrm{CH}_{2} \mathrm{C}_{3}\right) ;{ }^{13} \mathrm{C}-\mathrm{NMR} \delta 163(\mathrm{C}=\mathrm{O}), 154$ $(\mathrm{HC}=), 135,134,128(\mathrm{Ph}), 116(\mathrm{CN}), 103(\mathrm{C}=), 66\left(\mathrm{OCL}_{2} \mathrm{CH}_{2}\right), 22\left(\mathrm{OCH}_{2} \underline{\underline{C}} \mathrm{H}_{2}\right), 10$ $\left(\mathrm{OCH}_{2} \mathrm{CH}_{2} \underline{\mathrm{CH}_{3}}\right) ; \mathrm{IR}\left(\mathrm{cm}^{-1}\right)$ : 3078-2789 (m, C-H), 2226 (m, CN), 1719 (s, C=O), 1611 (s, $\mathrm{C}=\mathrm{C}), 1271,12210$ (s, C-O-C), 922, 810 (s, C-H out of plane). Anal. Calcd. for $\mathrm{C}_{13} \mathrm{H}_{11} \mathrm{Cl}_{2} \mathrm{NO}_{2}$ : C, 54.95; H, 3.90; N, 4.93; Found: C, 54.84; H, 3.97; N, 4.89.

\subsection{Propyl 2-cyano-3-(3,4-difluorophenyl)-2-propenoate}

Yield 46\%; mp 74 ${ }^{\circ} \mathrm{C},{ }^{1} \mathrm{H}-\mathrm{NMR} \delta 8.1$ (s, 1H, $\left.\mathrm{CH}=\right), 7.9-7.0(\mathrm{~m}, 3 \mathrm{H}, \mathrm{Ph}), 4.3(\mathrm{t}, 2 \mathrm{H}$, $\left.\mathrm{OCH}_{2}\right), 1.7\left(\mathrm{~m}, 2 \mathrm{H}, \mathrm{OCH}_{2} \underline{\mathrm{C}}_{2}\right), 1.0\left(\mathrm{t}, 3 \mathrm{H}, \mathrm{OCH}_{2} \mathrm{CH}_{2} \underline{\mathrm{C}}_{3}\right) ;{ }^{13} \mathrm{C}-\mathrm{NMR} \delta 163(\mathrm{C}=\mathrm{O}), 153$ $(\mathrm{HC}=), 150,130,125,118(\mathrm{Ph}), 116(\mathrm{CN}), 109(\mathrm{C}=), 67\left(\mathrm{OCH}_{2}\right), 22\left(\mathrm{OCH}_{2} \underline{\mathrm{CH}_{2}}\right), 10$ 
$\left(\mathrm{OCH}_{2} \mathrm{CH}_{2} \mathrm{CH}_{3}\right)$; IR (cm $\left.{ }^{-1}\right)$ : 3064-2809 (m, C-H), 2228 (m, CN), 1747 (s, C=O), 1597 (s, $\mathrm{C}=\mathrm{C}$ ), 1278 (s, C-O-C), 844, 761 (s, C-H out of plane). Anal. Calcd. for $\mathrm{C}_{13} \mathrm{H}_{11} \mathrm{~F}_{2} \mathrm{NO}_{2}$ : C, 62.15; H, 4.41; N, 5.58; Found: C, 61.97; H, 4.62; N, 5.54.

\subsection{Propyl 2-cyano-3-(3,5-difluorophenyl)-2-propenoate}

Yield $82 \%$; mp $76^{\circ} \mathrm{C},{ }^{1} \mathrm{H}-\mathrm{NMR} \delta 8.1(\mathrm{~s}, 1 \mathrm{H}, \mathrm{CH}=), 7.7-6.7(\mathrm{~m}, 3 \mathrm{H}, \mathrm{Ph}), 4.3(\mathrm{t}, 2 \mathrm{H}$,

$\left.\mathrm{OCH}_{2}\right), 1.8\left(\mathrm{~m}, 2 \mathrm{H}, \mathrm{OCH}_{2} \mathrm{CH}_{2}\right), 1.0\left(\mathrm{t}, 3 \mathrm{H}, \mathrm{OCH}_{2} \mathrm{CH}_{2} \mathrm{CH}_{3}\right) ;{ }^{13} \mathrm{C}-\mathrm{NMR} \delta 162(\mathrm{C}=\mathrm{O}), 153$ $(\mathrm{HC}=), 163,145,135(\mathrm{Ph}), 115(\mathrm{CN}), 103(\mathrm{C}=), 67\left(\mathrm{OCH}_{2}\right), 22\left(\mathrm{OCH}_{2} \underline{C H}_{2}\right), 10$ $\left(\mathrm{OCH}_{2} \mathrm{CH}_{2} \underline{\mathrm{CH}}_{3}\right) ; \mathrm{IR}\left(\mathrm{cm}^{-1}\right)$ : 3127-2776 (m, C-H), 2228 (m, CN), 1740 (s, C=O), 1618 (s, $\mathrm{C}=\mathrm{C}$ ), 1244 (s, C-O-C), 839, 767 (s, C-H out of plane). Anal. Calcd. for $\mathrm{C}_{13} \mathrm{H}_{11} \mathrm{~F}_{2} \mathrm{NO}_{2}$ : C, 62.15; H, 4.41; N, 5.58; Found: C, 61.25; H, 4.40; N, 5.79.

\subsection{Propyl 2-cyano-3-(2-chloro-4-fluorophenyl)-2-propenoate}

Yield $88 \%$; mp $80^{\circ} \mathrm{C},{ }^{1} \mathrm{H}-\mathrm{NMR} \delta 8.1$ (s, $\left.1 \mathrm{H}, \mathrm{CH}=\right), 7.9-6.7(\mathrm{~m}, 3 \mathrm{H}, \mathrm{Ph}), 4.3(\mathrm{t}, 2 \mathrm{H}$, $\left.\mathrm{OCH}_{2}\right), 1.7\left(\mathrm{~m}, 2 \mathrm{H}, \mathrm{OCH}_{2} \mathrm{CH}_{2}\right), 1.0\left(\mathrm{t}, 3 \mathrm{H}, \mathrm{OCH}_{2} \mathrm{CH}_{2} \underline{\mathrm{CH}}_{3}\right) ;{ }^{13} \mathrm{C}-\mathrm{NMR} \delta 164(\mathrm{C}=\mathrm{O}), 152$ $(\mathrm{HC}=), 163,156,132,113(\mathrm{Ph}), 115(\mathrm{CN}), 99(\mathrm{C}=), 67\left(\mathrm{OCH}_{2}\right), 21\left(\mathrm{OCH}_{2} \underline{C H}_{2}\right), 10$ $\left(\mathrm{OCH}_{2} \mathrm{CH}_{2} \underline{\mathrm{CH}}_{3}\right) ; \mathrm{IR}\left(\mathrm{cm}^{-1}\right)$ : 3143-2798 (m, C-H), 2221 (m, CN), 1721 (s, C=O), 1627 (s, $\mathrm{C}=\mathrm{C}$ ), 1276 (s, C-O-C), 877, 734 (s, C-H out of plane). Anal. Calcd. for $\mathrm{C}_{13} \mathrm{H}_{11} \mathrm{ClFNO}_{2}$ :

C, 58.33; H, 4.14; N, 5.23; Found: C, 59.46; H, 4.35; N, 5.58.

\subsection{Propyl 2-cyano-3-(2-chloro-6-fluorophenyl)-2-propenoate}

Yield 68\%; mp 50 ${ }^{\circ} \mathrm{C},{ }^{1} \mathrm{H}-\mathrm{NMR} \delta 8.1(\mathrm{~s}, 1 \mathrm{H}, \mathrm{CH}=), 7.4-6.7(\mathrm{~m}, 3 \mathrm{H}, \mathrm{Ph}), 4.3(\mathrm{t}, 2 \mathrm{H}$, $\left.\mathrm{OCH}_{2}\right), 1.7\left(\mathrm{~m}, 2 \mathrm{H}, \mathrm{OCH}_{2} \mathrm{CH}_{2}\right), 1.0\left(\mathrm{t}, 3 \mathrm{H}, \mathrm{OCH}_{2} \mathrm{CH}_{2} \mathrm{C}_{3}\right) ;{ }^{13} \mathrm{C}-\mathrm{NMR} \delta 164(\mathrm{C}=\mathrm{O}), 152$ $(\mathrm{HC}=), 163,153,130,126,119,116(\mathrm{Ph}), 115(\mathrm{CN}), 92(\mathrm{C}=), 67\left(\mathrm{OCH}_{2}\right), 21$ 
$\left(\mathrm{OCH}_{2} \underline{\mathrm{CH}}_{2}\right), 10\left(\mathrm{OCH}_{2} \mathrm{CH}_{2} \underline{\mathrm{CH}}_{3}\right) ; \mathrm{IR}\left(\mathrm{cm}^{-1}\right):$ 3165-2813 (m, C-H), $2228(\mathrm{~m}, \mathrm{CN}), 1751$ (s, $\mathrm{C}=\mathrm{O}$ ), 1617 (s, C=C), 1287 (s, C-O-C), 812, 765 (s, C-H out of plane). Anal. Calcd. for $\mathrm{C}_{13} \mathrm{H}_{11} \mathrm{ClFNO}_{2}: \mathrm{C}, 58.33 ; \mathrm{H}, 4.14 ; \mathrm{N}, 5.23$; Found: C, 54.76; H, 4.65; N, 5.63.

\subsection{Propyl 2-cyano-3-(3-chloro-2-fluorophenyl)-2-propenoate}

Yield 73\%; mp 53 ${ }^{\circ} \mathrm{C},{ }^{1} \mathrm{H}-\mathrm{NMR} \delta 8.5(\mathrm{~s}, 1 \mathrm{H}, \mathrm{CH}=), 8.3-7.2(\mathrm{~m}, 3 \mathrm{H}, \mathrm{Ph}), 4.3(\mathrm{t}, 2 \mathrm{H}$,

$\left.\mathrm{OCH}_{2}\right), 1.7\left(\mathrm{~m}, 2 \mathrm{H}, \mathrm{OCH}_{2} \mathrm{CH}_{2}\right), 1.0\left(\mathrm{t}, 3 \mathrm{H}, \mathrm{OCH}_{2} \mathrm{CH}_{2} \mathrm{CH}_{3}\right) ;{ }^{13} \mathrm{C}-\mathrm{NMR} \delta 163(\mathrm{C}=\mathrm{O}), 152$ $(\mathrm{HC}=), 162,153,130,126,119,116(\mathrm{Ph}), 115(\mathrm{CN}), 92(\mathrm{C}=), 67\left(\mathrm{OCH}_{2}\right), 21$

$\left(\mathrm{OCH}_{2} \underline{\mathrm{CH}_{2}}\right), 10\left(\mathrm{OCH}_{2} \mathrm{CH}_{2} \underline{\mathrm{CH}}_{3}\right) ; \mathrm{IR}\left(\mathrm{cm}^{-1}\right):$ 3045-2767 (m, C-H), 2224 (m, CN), 1739 (s, $\mathrm{C}=\mathrm{O}$ ), 1614 (s, C=C), 1265 (s, C-O-C), 766719 (s, C-H out of plane). Anal. Calcd. for $\mathrm{C}_{13} \mathrm{H}_{11} \mathrm{ClFNO}_{2}$ : C, 58.33; H, 4.14; N, 5.23; Found: C, 58.17; H, 4.21; N, 5.25.

\subsection{Propyl 2-cyano-3-(3-chloro-4-fluorophenyl)-2-propenoate}

Yield $84 \%$; mp $118^{\circ} \mathrm{C},{ }^{1} \mathrm{H}-\mathrm{NMR} \delta 8.2(\mathrm{~s}, 1 \mathrm{H}, \mathrm{CH}=), 8.1-7.2(\mathrm{~m}, 3 \mathrm{H}, \mathrm{Ph}), 4.3(\mathrm{t}, 2 \mathrm{H}$, $\left.\mathrm{OCH}_{2}\right), 1.7\left(\mathrm{~m}, 2 \mathrm{H}, \mathrm{OCH}_{2} \mathrm{CH}_{2}\right), 1.0\left(\mathrm{t}, 3 \mathrm{H}, \mathrm{OCH}_{2} \mathrm{CH}_{2} \mathrm{CH}_{3}\right) ;{ }^{13} \mathrm{C}-\mathrm{NMR} \delta 163(\mathrm{C}=\mathrm{O}), 154$ $(\mathrm{HC}=), 153,130,128,120,117(\mathrm{Ph}), 115(\mathrm{CN}), 92(\mathrm{C}=), 67\left(\mathrm{OCH}_{2}\right), 21\left(\mathrm{OCH}_{2} \underline{\mathrm{CH}}_{2}\right), 10$ $\left(\mathrm{OCH}_{2} \mathrm{CH}_{2} \underline{\mathrm{CH}}_{3}\right) ; \mathrm{IR}\left(\mathrm{cm}^{-1}\right): 3187-2870(\mathrm{~m}, \mathrm{C}-\mathrm{H}), 2226(\mathrm{~m}, \mathrm{CN}), 1714$ (s, C=O), 1616 (s, $\mathrm{C}=\mathrm{C}$ ), 1258 (s, C-O-C), 829, 761 (s, C-H out of plane). Anal. Calcd. for $\mathrm{C}_{13} \mathrm{H}_{11} \mathrm{ClFNO}_{2}$ : C, 58.33; H, 4.14; N, 5.23; Found: C, 58.18; H, 4.12; N, 5.10.

\section{Copolymerization}

Copolymers of the ST and the PCPP monomers were prepared in 25-mL glass screw cap vials at $\mathrm{ST} / \mathrm{PCPP}=3(\mathrm{~mol})$ the monomer feed using $0.12 \mathrm{~mol} / \mathrm{L}$ of $\mathrm{ABCN}$ at an overall monomer concentration $2.44 \mathrm{~mol} / \mathrm{L}$ in $10 \mathrm{~mL}$ of toluene. The copolymerization was 
conducted at $70^{\circ} \mathrm{C}$. After a predetermined time, the mixture was cooled to room temperature, and precipitated dropwise in methanol. The composition of the copolymers was determined based on the nitrogen content.

Copolymerization (Sch. 1) of ST and the ring-disubstituted PCPP resulted in formation of copolymers (Table 1) with weight-average molecular masses 11.0 to $48.1 \mathrm{kD}$.<smiles>[R]c1ccccc1/C=C(\N)C(=O)OCC</smiles>

Sch. 1. ST-PCPP statistical copolymer synthesis, $\mathrm{R}=$ 2-fluoro-5-methyl, 3-iodo-4methoxy, 5-iodo-2-methoxy, 3,5-dichloro, 3,4-difluoro, 3,5-difluoro, 2-chloro-4-fluoro, 2chloro-6-fluoro, 3-chloro-2-fluoro, and 3-chloro-4-fluoro.

According to elemental analysis, between 18.3 and $42.6 \mathrm{~mol} \%$ of TSE monomer is present in the copolymers prepared at $\mathrm{ST} / \mathrm{PCPP}=3(\mathrm{~mol})$, which is indicative of relatively high reactivity of the monomers towards ST. 
Table 1. Copolymerization of Styrene $\left[\mathrm{M}_{1}\right]$ and Ring-disubstituted propyl 2-cyano-3phenyl-2-propenoates, $\mathrm{RPhCH}=\mathrm{C}(\mathrm{CN}) \mathrm{CO}_{2} \mathrm{C}_{3} \mathrm{H}_{7}\left[\mathrm{M}_{2}\right]$.

\begin{tabular}{|c|c|c|c|c|c|c|c|c|c|}
\hline & & & & & & \multicolumn{4}{|c|}{$T G A$} \\
\hline $\mathrm{R}$ & $\begin{array}{l}\text { Yield }^{a} \\
\text { (wt\%) }\end{array}$ & $\underset{(\mathrm{wt} \%)}{\mathrm{N}}$ & $\begin{array}{c}\mathrm{m}_{2} \text { in } \\
\text { copol. } \\
\text { (mol } \\
\% \text { ) }\end{array}$ & $\begin{array}{l}\mathrm{M}_{\mathrm{W}} \\
(\mathrm{kD})\end{array}$ & $\begin{array}{c}\mathrm{T}_{\mathrm{g}} \\
\left({ }^{\circ} \mathrm{C}\right)\end{array}$ & $\begin{array}{c}\text { Onset of } \\
\text { decomp. } \\
\left({ }^{\circ} \mathrm{C}\right)\end{array}$ & $\begin{array}{c}10 \\
\text { wt } \% \\
\text { loss } \\
\left({ }^{\circ} \mathrm{C}\right)\end{array}$ & $\begin{array}{l}50 \\
\text { wt } \% \\
\text { loss } \\
\left({ }^{\circ} \mathrm{C}\right)\end{array}$ & $\begin{array}{c}\text { Residue } \\
\text { wt } \%\end{array}$ \\
\hline 2-F-5-CH & 18.3 & 2.78 & 28.8 & 26.3 & 124 & 255 & 302 & 336 & 2 \\
\hline $3-\mathrm{I}-4-\mathrm{OCH}_{3}$ & 12.8 & 1.85 & 21.2 & 23.9 & 103 & 135 & 277 & 338 & 2 \\
\hline $5-\mathrm{I}-2-\mathrm{OCH}_{3}$ & 15.8 & 2.17 & 27.5 & 11.0 & 111 & 207 & 303 & 340 & 3 \\
\hline $3,5-\mathrm{Cl}_{2}$ & 17.6 & 2.69 & 30.5 & 48.1 & 151 & 252 & 290 & 327 & 2 \\
\hline $3,4-\mathrm{F}_{2}$ & 14.7 & 2.84 & 30.1 & 36.2 & 138 & 171 & 299 & 331 & 1 \\
\hline $3,5-\mathrm{F}_{2}$ & 17.6 & 2.67 & 27.6 & 32.1 & 112 & 205 & 275 & 345 & 2 \\
\hline 2-Cl-4-F & 13.8 & 2.53 & 27.9 & 29.3 & 121 & 207 & 313 & 349 & 3 \\
\hline 2-Cl-6-F & 12.2 & 1.71 & 15.9 & 27.9 & 112 & 203 & 312 & 361 & 2 \\
\hline $3-\mathrm{Cl}-2-\mathrm{F}$ & 17.8 & 2.67 & 28.9 & 30.2 & 112 & 210 & 322 & 369 & 1 \\
\hline 3-Cl-4-F & 12.2 & 2.65 & 28.5 & 28.1 & 110 & 190 & 323 & 362 & 1 \\
\hline
\end{tabular}

aPolymerization time was $8 \mathrm{~h}$.

\section{Structure and thermal properties}

The structure of ST-PCPP copolymers was characterized by IR and NMR spectroscopy. A comparison of the spectra of the monomers, copolymers and polystyrene shows, that the 
reaction between the monomers and ST is a copolymerization. IR spectra of the copolymers show overlapping bands in $3200-2820 \mathrm{~cm}^{-1}$ region corresponding to $\mathrm{C}-\mathrm{H}$ stretch vibrations. The bands for the PCPP monomer unit are 2246-2238 (w, CN), 1752-1733 (s, C=O), and $1252-1226 \mathrm{~cm}^{-1}(\mathrm{~m}, \mathrm{C}-\mathrm{O})$. Benzene rings of both monomers show ring stretching bands at $1500-1400 \mathrm{~cm}^{-1}$ as well as a doublet $824-715 \mathrm{~cm}^{-1}$, associated with C-H out of plane deformations. These bands can be readily identified in styrene copolymers with PCPP monomers containing cyano and carbonyl electron withdrawing groups [20].

The ${ }^{1} \mathrm{H}-\mathrm{NMR}$ spectra of the ST-PCPP copolymers show a broad double peak in a 6.0-8.0 ppm region corresponding to phenyl ring protons. A resonance at 4.5-3.7 ppm is assigned to the methyleneoxy protons of PCPP monomer unit. A broad resonance at 3.0-2.0 ppm is assigned to the methine protons of PCPP, and methine and methylene protons of ST monomer unit close to the propenoate unit, which are more subjected to deshielding than the ones in polystyrene. A broad resonance peak in $0.8-2.3 \mathrm{ppm}$ range is attributed to the methine and methylene protons of styrene monomer sequences, as well as to alkyl ester protons of PCPP. The ${ }^{13} \mathrm{C}-\mathrm{NMR}$ spectra also support the suggested skeletal structure of the copolymers. Thus, the assignment of the peaks is as follows: 164-162 ppm $(\mathrm{C}=\mathrm{O}), 154-131$ ppm (quaternary carbons of both phenyls), 146-122 ppm (phenyl carbons), 117-113 ppm (CN), 62-51 ppm (methine, quaternary carbons and alkoxy PCPP carbons), 48-41 ppm (ST methine), and 43-40 ppm (ST methylene) carbons of PCPP. The IR and NMR data showed that these are true copolymers, composed of both TSE and ST monomer units similarly to reported earlier copolymers [20]. 
The copolymers prepared in the present work are all soluble in ethyl acetate, THF, DMF and $\mathrm{CHCl}_{3}$ and insoluble in methanol, ethyl ether, and petroleum ether. They are amorphous and show no crystalline DSC endotherm. Results of thermal analysis of STPCPP copolymers are presented in Table 1 . Information on the degradation of the copolymers was obtained from thermogravimetric analysis. Decomposition of the copolymers in nitrogen occurred in two steps, first in the $200-500^{\circ} \mathrm{C}$ range with residue (1$3 \% \mathrm{wt}$ ), which then decomposed in the $500-800^{\circ} \mathrm{C}$ range. The decomposition products were not analyzed in this study, and the mechanism has yet to be investigated.

\section{Conclusions}

Novel trisubstituted ethylenes, ring-disubstituted propyl 2-cyano-3-phenyl-2-propenoates were prepared and copolymerized with styrene. The compositions of the copolymers were calculated from nitrogen analysis and the structures were analyzed by IR, $\mathrm{H}^{1}$ and ${ }^{13} \mathrm{C}-\mathrm{NMR}$. The thermal gravimetric analysis indicated that the copolymers decompose in in two steps, first in the $200-500^{\circ} \mathrm{C}$ range with residue (1-3\%wt), which then decomposed in the $500-800^{\circ} \mathrm{C}$ range.

\section{Funding}

The authors are grateful to acknowledge that the project was partly supported by the Coatings Industry Education Fund (CIEF) and Chicago Society of Coating Technology (CSCT). 


\section{References}

[1] Stereoselective cascade assembling of benzylidenecyanoacetates and 1,3dimethylbarbituric acid into (1R*,2S*)-1-cyano-5,7-dialkyl-4,6,8-trioxo-2-aryl-5,7diazaspiro[2.5] octane-1-carboxylates. Elinson, Michail N.; Vereshchagin, Anatoly N.; Korshunov, Alexander D.; Ryzhkov, Fedor V.; Egorov, Mikhail P. Heterocyclic Communications (2017), 23(2), 85-90.

[2] Polysiloxane-Supported NAD(P)H Model 1-Benzyl-1,4-dihydronicotinamide: Synthesis and Application in the Reduction of Activated Olefins. Zhang, Baolian; Zhu, XiaoQing; Lu, Jin-Yong; He, Jiaqi; Wang, Peng G.; Cheng, Jin-Pei. Journal of Organic Chemistry (2003), 68(8), 3295-3298.

[3] Selective hydrolysis of 1-cyanocyclopropane-1-carboxylates: concise preparation of 1carbamoylcyclopropane-1-carboxylates. Liu, Jiaming; Zhang, Feixiang; Wang, Ting; Qing, Xushun; Wang, Cunde. Journal of Chemical Research (2016), 40(11), 694-697.

[4] Reaction products of indandione with ethyl $\alpha$-cyano- $\beta$-arylacrylates. Diurno, M. V.; Cirino, G.; Squassi, A. Bollettino - Societa Italiana di Biologia Sperimentale (1984), 60(1), 79-84.

[5] Base-free Knoevenagel condensation catalyzed by copper metal surfaces. Schneider, E. M.; Zeltner, M.; Kranzlin, N.; Grass, R. N.; Stark, W. J. Chemical Communications (Cambridge, United Kingdom) (2015), 51(53), 10695-10698.

[6] Selective hydrolysis of 1-cyanocyclopropane-1-carboxylates: concise preparation of 1carbamoylcyclopropane-1-carboxylates. Liu, Jiaming; Zhang, Feixiang; Wang, Ting; Qing, Xushun; Wang, Cunde. Journal of Chemical Research (2016), 40(11), 694-697. 
[7] Preparation of a novel Fe3O4@SiO2@propyl@DBU magnetic core-shell nanocatalyst for Knoevenagel reaction in aqueous medium. Zhang, Xiaoyu; He, Xiaoyan; Zhao, Sanhu. Green Chemistry Letters and Reviews (2021), 14(1), 85-98.

[8] A highly efficient protocol for the regio- and stereo-selective synthesis of spiro pyrrolidine and pyrrolizidine derivatives by multicomponent reaction. Dandia, Anshu; Jain, Anuj K.; Laxkar, Ashok K.; Bhati, Dharmendra S. Tetrahedron Letters (2013), 54(24), 3180-3184.

[9] A PEG bridged tertiary amine functionalized ionic liquid exhibiting thermoregulated reversible biphasic behavior with cyclohexane/isopropanol: synthesis and application in Knoevenagel condensation. Luo, Jun; Xin, Tantan; Wang, Yinglei. New Journal of Chemistry (2013), 37(2), 269-273.

[10] Synthesis of 5,6-dichloroindan-1-acids and their tetrazolyl derivatives as analgesic and anti-inflammatory agents. Pal, Rajib Kumar; Yasmin, Hasina; Nahar, Lutfun; Datta, Bidyut Kanti; Chowdhury, Abul Kalam Azad; Kundu, Joydeb Kumar; Bachar, Sitesh Chandra; Sarker, Satyajit Dey. Medicinal Chemistry (2012), 8(5), 874-882.

[11] $\beta$-Amino- $\beta$-(pyrid-4-yl)acrylonitrile in heterocyclic synthesis: synthesis of some new pyridine, pyridone, pyrazole, thiophene, fused pyrimidine and triazine derivatives. Ghozlan, Said A. S.; Hassanien, Abu Zeid A. Tetrahedron (2002), 58(46), 9423-9429.

[12] Synthesis and histamine $\mathrm{H} 2$ agonistic activity of arpromidine analogs: replacement of the pheniramine-like moiety by non-heterocyclic groups. Buschauer, A.; Friese-Kimmel, A.; 
Baumann, G.; Schunack, W. European Journal of Medicinal Chemistry (1992), 27(4), 321-

30.

13. Park, K. H., Twieg, R. J., Ravikiran, R., Rhodes, L. F, Shick, R. A., Yankelevich, D., Knoesen, A. (2004) Macromolecules, 37(14), 5163.

14. Elinson, M.N., Vereshchagin, A.N., Korshunov, A.D., Ryzhkov, F.V., Egorov, M.P., (2017) Heterocyclic Communications, 23(2), 85-90.

15. Zhang, B., Zhu, X-Q., Lu, J-Y, He, J., Wang, P.G, Cheng, J.-P. (2003) J. Org. Chem. 68(8), 3295-3298.

16. Gan, Z-J, Wang, Y-H, Xu, Y-G, Guo, T., Wang, J., Song, Q., Xu, X.-J., Hu, S-Y, Wang, Y-J, Wang, D-C., et al. (2015) Organic \& Biomolecular Chem., 13(20), 56565673.

17. Das, S., Yasmin, H., Mehedi, M.M., Roy, S.C., Nahar, L., Mukhlesur, R.M., Gibbons, S., Bachar, S.C., Sarker, S.D. (2008) Tetrahedron, 64(37), 8642-8645.

18. Zhu, X-Q, Zou, H-L, Yuan, P-W, Liu, Y., Cao, L., Cheng, J-P. (2000) Perkin 2, (9), $1857-1861$.

19. SciFinder Structure Search January 7, 2022.

20. Novel Copolymers of Styrene. 1. Alkyl Ring-Substituted Propyl 2-Cyano-3-Phenyl-2Propenoates. G.B. Kharas, A.M. Claro, Y. Gao, J.S. Bhanot, P. Bosek, Z. Carwile, T.R. Corwin, C.J. Gideon, P.R. Fitzpatrick, I.M. Flores, and J.M. Rickter. J. Macromol. Sci. A53(10) 595-599 (2016). 
21. Smith, M. B., March, J. (2001) Addition to Carbon-Hetero Multiple Bonds, In March's Advanced Organic Chemistry; J. Wiley \& Sons: New York, Ch.16, 1225. 